\title{
Vaginal Birth after Cesarean Section vs Elective Repeat Cesarean Section: A Cost-consequence Analysis
}

\author{
Chanil D Ekanayake ${ }^{1}$, Swetha Thangasamy ${ }^{2}$, Arunasalam Pathmeswaran ${ }^{3}$, Rasika Herath ${ }^{4}$, Sanjeewa Padumadasa ${ }^{5}$, Sunil
} Fernando ${ }^{6}$, Tiran Dias $^{7}$, Prasantha Wijesinghe ${ }^{8}$

\begin{abstract}
Aim: There is limited evidence on the cost-effectiveness of vaginal birth after cesarean section vs elective repeat cesarean section. The aim of this study was to compare the outcomes and costs of vaginal birth after cesarean section vs elective repeat cesarean section.

Materials and methods: A cost-consequence study was done from April 1, 2018 to March 31, 2019. Women admitted for delivery with one previous cesarean section were selected. Exclusion criteria were: multiple gestations, placenta previa, morbidly adherent placenta, birth weight $<2.5 \mathrm{~kg}$ or $>4 \mathrm{~kg}$, and patients undergoing emergency cesarean section for antepartum emergencies. The outcomes and costs of vaginal birth after cesarean section vs elective repeat cesarean section were compared.

Results: The vaginal birth after cesarean section was successful in 34 (50.7\%) cases. There was no difference in median postpartum hospital stay between vaginal birth after cesarean section $(n=67)[2(1-3)$ days] and elective repeat cesarean section $(n=158)[2(2-2.25)$ days] $(p=0.15)$. There was no significant difference in total costs between vaginal birth after cesarean section and elective repeat cesarean section [USD 476 (420-566) vs USD 470 (452-494), $p=0.78]$.

The direct cost of the vaginal birth after cesarean section successful group $(n=34)$ was USD 427 (361-462) vs failed vaginal birth after cesarean section group $(n=32)$ USD $505(476-716)(p<0.001)$. There was a significant difference in postpartum hospital stay between successful vaginal birth after cesarean section [2 (1-3) days], failed vaginal birth after cesarean section [2 (2-3.75) days], and elective repeat cesarean section [2 (2-2.25) days] $(p<0.01)$.

Conclusion: The absence of a significant difference in postpartum hospital stay and cost between vaginal birth after cesarean section and elective repeat cesarean section raises serious clinical concerns with possible implications for health policy stakeholders.

Keywords: Cost-consequence analysis, Elective repeat cesarean section, Vaginal birth after cesarean section.

Journal of South Asian Federation of Obstetrics and Gynaecology (2021): 10.5005/jp-journals-10006-1849
\end{abstract}

\section{INTRODUCTION}

There has been an alarming rise in cesarean section rates worldwide. ${ }^{1-3}$ In Sri Lanka, the cesarean section rate has climbed from $9 \%$ in 1986 to $39.1 \%$ in $2018 .{ }^{4}$ This may be due to a multitude of reasons; use of intrapartum electronic fetal monitoring, changes in obstetric training, medicolegal concerns, alterations in social expectations of pregnancy outcome, and maternal autonomy in decision making. ${ }^{5-9}$

The rising cesarean section rates have had a "snowball" effect resulting in a significant amount of women with a previous cesarean section for which the delivery options are either an elective repeat cesarean section (ERCS) or vaginal birth after cesarean section (VBAC).

VBAC is associated with reduced morbidity and a lower risk of complications in future pregnancies. ${ }^{10,11}$ Despite VBAC being considered a viable and safe alternative to ERCS for women with one previous cesarean section, a significant proportion of women undergo ERCS. ${ }^{10,11}$ Local and international evidence shows that the previous cesarean section was found to be the leading cause of ERCS. ${ }^{12,13}$ Nonetheless, there are only a handful of articles on the cost-effectiveness of VBAC vs ERCS, with almost no data from low resource settings. ${ }^{14-16}$

Improvements in health care and social pressure from a burgeoning middle-class are probable reasons for the rising cesarean section rate in Sri Lanka. The fact that it is also a low resource setting with a policy of free public health further emphasizes the importance of studying VBAC vs ERCS from a
1,2 Department of Clinical Sciences, Faculty of Medicine, General Sir John Kotelawala Defence University, Colombo, Western Province, Sri Lanka

${ }^{3}$ Department of Public Health, University of Kelaniya, Ragama, Western Province, Sri Lanka

${ }^{4-8}$ Department of Obstetrics and Gynaecology, University of Kelaniya, Ragama, Western province, Sri Lanka

Corresponding Author: Chanil D Ekanayake, Department of Clinical Sciences, Faculty of Medicine, General Sir John Kotelawala Defence University, Colombo, Western Province, Sri Lanka, Phone: +94 713283668, e-mail: cdekanayake2000@yahoo.co.uk

How to cite this article: Ekanayake CD, Thangasamy $S$, Pathmeswaran A, et al. Vaginal Birth after Cesarean Section vs Elective Repeat Cesarean Section: A Cost-consequence Analysis. J South Asian Feder Obst Gynae 2021;13(1):38-43.

Source of support: Nil

Conflict of interest: None

provider's perspective.Therefore, a cost-consequence study comparing the outcomes and costs of VBAC vs ERCS was designed.

\section{Materials and Methods}

A retrospective observational study was done in the professorial obstetrics and gynecology unit of the North Colombo Teaching Hospital (NCTH), Ragama. Women with one previous cesarean section who were admitted for delivery at NCTH, Ragama from April 1, 2018 to March 31, 2019 were included. Exclusion criteria were; 
multiple gestations, placenta previa, morbidly adherent placenta, birth weight $<2.5 \mathrm{~kg}$ or $>4 \mathrm{~kg}$, and patients undergoing emergency cesarean section for antepartum emergencies.

The primary outcome to calculate sample size was the postpartum hospital stay. The mean postpartum hospital stay (standard deviation, SD) for ERCS and VBAC were 2.57 (0.52) and 2.82 (0.49) days based on a pilot study done on a subset of women in the unit. A difference of 0.5 postpartum days was considered as a significant difference. A power of $80 \%$ and a type 1 error of 0.05 with patient allocation at a ratio of 2:1 for ERCS and VBAC required 97 and 49 patients, respectively to detect a 0.5-day difference.

The birth register was evaluated initially to identify eligible patients and data were collected from their in-patient hospital records. The primary outcome was a postpartum hospital stay. The other secondary outcomes were; maternal and neonatal complications, cost of postpartum hospital stay, and cost of neonatal intensive care. Details of age, body mass index (BMI), parity, birth weight, mode of delivery hospital stay, appearance, pulse, grimace, activity, and respiration (APGAR) scores (5 min), maternal and neonatal complications were obtained from the hospital clinical records.

Costing of direct healthcare costs (labor, investigations, equipment, drugs, and utility costs) were calculated based on the method illustrated by Ekanayake et al. ${ }^{17}$ A time-driven, activity-based costing method was adopted to calculate the cost of wages. Therefore, the wage cost per minute for each category of staff was calculated and used to cost the various activities based on the amount of time spent. The cost of equipment and drugs were obtained from the medical supplies division of the Ministry of Health, Sri Lanka. The cost of equipment per patient was calculated assuming it lasts for 10 -years. Investigation costs included laboratory and imaging costs for pre- and postoperative period. The cost for a particular test considered the equipment cost, labor cost, and reagent cost. The labor cost per test was calculated using the laboratory technician's labor cost per minute and time spent per test. The reagent cost per test was based on the number of tests per year and the amount of reagents used for that time period. The cost of drugs was obtained from the medical supplies division of the Ministry of Health, Sri Lanka. Utility costs were calculated per patient per day for space, water, electricity, food, administrative, cleaning services, telephone services, and security costs. The rental value for the ward space was obtained from the National Housing Development Authority. The other utilities were calculated using the respective monthly bills divided by the average midnight total of patients to obtain the cost of a particular utility per patient per day [e.g., electricity cost]. The cost of food obtained from the hospital was calculated considering the food cost and labor. The total utility cost per patient per day was obtained including the sum of all the above sub-categories.
Maternal costs included labor, investigations, equipment, drugs, and utility costs. Neonatal costs incorporated the sum of the costs for newborn intensive care unit (NICU) stay, phototherapy, neonatal investigations, and medications. Total cost was considered as the sum of maternal and neonatal costs.

Non-tradable goods (labor and utilities) were calculated using purchasing power parity (PPP) factor of 49.50 for $2018 .{ }^{18}$ The exchange rate (1 USD $=182.73$ LKR) was used for equipment, drugs, and investigations, which were tradable commodities.

The variables that met the criteria for normality were assessed using Student's t-test whilst the ones that failed to meet the criteria for normality were assessed using the Mann-Whitney $U$ test to compare the differences in outcomes and costs between the two groups, VBAC and ERCS. Analysis was by intention to treat. The VBAC group was separated into VBAC-successful and VBAC-failed and compared with the ERCS group using the Kruskal-Wallis test. Complications between the groups were compared using the Chi-square test and Fisher's exact test. Samples of the same size as the data were drawn after specifying the number of successes for the VBAC group and the difference between the median of the two groups was calculatedto find out the cost at which VBAC equaled ERCS with a 95\% degree of confidence. SPSS statistics 20.0 was used for statistical analyses.

\section{Results}

The basic characteristics of the study population are shown in Table 1. The VBAC success rate was $50.7 \%$ with 34 successful cases including seven instrumental deliveries. The median postpartum hospital stay was 2 with an interquartile range of (1-3) and (2-2.25) days, for VBAC and ERCS, respectively ( $p=0.15$, Table 2 . There was no apparent difference in maternal complications (Table 2).

Neonatal complications were significantly more in the ERCS group (Table 2). There were 5(7.9\%) and 14 (8.9\%) cases of NICU admissions in VBAC and ERCS groups, respectively. NICU admissions in the VBAC-successful and VBAC-failed group were 3 (8.6\%) and 2 (9.4\%), respectively. Neonatal complications; fever and jaundice were also more common in the ERCS group. There was one baby with meconium aspiration in a failed VBAC. There were no cases of hypoxic-ischemic encephalopathy (HIE). APGAR scores (5 min) were 10 with an interquartile range of (10-10) for both categories.

The material costs were similar between the two groups with USD 467 (419-522) vs USD 459 (452-486) between VBAC and ERCS groups, respectively $(p=0.74)$. There was no significant difference in neonatal costs between the two groups $(p=0.31)$. There was no significant difference in total costs between the two groups with $476(420-566)$ vs $470(452-494)$ between VBAC and ERCS groups ( $p=0.78$, Table 3).

Subgroup analysis of successful and failed VBAC showed that the VBAC-successful group (VBAC success rate 100\%) and failed VBAC group (VBAC success rate $0 \%$ ) had maternal costs of USD 427 (361-462) vs USD 505 (476-716), respectively as opposed to USD 459

Table 1: Basic characteristics of the study population

\begin{tabular}{lccc}
\hline & $\operatorname{VBAC}(n=67)$ & ERCS $(n=158)$ & Significance $(p)$ \\
\hline Age (years) (mean, SD) & $28.2(2.86)$ & $27.5(2.52)$ & $0.06^{\#}$ \\
BMI (kg/m²) (mean, SD) & $25.79(2.16)$ & $25.93(1.81)$ & $0.60^{\#}$ \\
Parity [median, (IQ1-IQ3)] & $2.00(2.00-2.00)$ & $2.00(2.00-2.00)$ & $0.04^{*}$ \\
Birth weight (kg) (mean, SD) & $3.03(0.58)$ & $3.10(0.42)$ & $0.32^{*}$ \\
\hline
\end{tabular}

*Mann-Whitney U test; \#Student's t-test; VBAC, vaginal birth after cesarean section;

ERCS, elective repeat cesarean section; SD, standard deviation, BMI, body mass index 
(452-486) in the ERCS group ( $p<0.001$, Tables 4 and 5. There was a significant difference in total costs between successful VBAC [USD 427 (361-483)], failed VBAC [USD 519, (476-809)] and ERCS groups [USD 470 (452-292)] ( $p<0.001$, Table 5. Less than $5 \%$ of the 100000 simulations resulted in the overall VBAC median cost being higher than the ERCS median cost when the VBAC success rate was $80 \%$ (52 out of 65 ).

\section{Discussion}

To our knowledge, this is the first attempt at comparing both outcomes and costs of VBAC Vs ERCS in a low resource setting. The main findings of the study were that there was no difference in costs, postpartum hospital stay, and maternal complications between ERCS and VBAC. There were more neonatal complications in the ERCS group.

Evidence from literature shows that VBAC was associated with a lower cost. ${ }^{19}$ There is further evidence to show that VBAC was comparatively more cost-effective compared to ERCS. ${ }^{14,15}$ The probable reason for the contradictory finding in our studymay bethe relatively low VBAC success rate of $50.7 \%$. The study finding of no significant difference in hospital stay also contradicts evidence from literature which shows that postpartum hospital stay was also shorter in VBAC patients compared to ERCS. ${ }^{20,21}$ The reason for this may be the fact that hospital stay depends more on individual practice rather than complications. ${ }^{22}$ Our findings of comparable maternal outcomes between the two groups are corroborated by evidence from India and Iran. ${ }^{21,23}$ Although these studies show comparable neonatal outcomes between VBAC and ERCS, in-depth analysis shows that ERCS is associated with higher respiratory morbidity whilst VBAC was found to have a slightly higher risk of perinatal death and HIE. ${ }^{10,21,23}$ Although there were no cases of perinatal deaths there was one case of meconium aspiration in the VBAC group. However, unexpectedly there were more neonates with fever and jaundice in the ERCS group.

Maternal and neonatal complications are not common. This study was not powered to detect a statistical significance between individual maternal and neonatal complications as the sample size was small. Therefore, complications were reported with percentages. To overcome this limitation maternal and neonatal complications were pooled together, i.e., all the patients with maternal complications were considered together and analyzed.

This study only considered clinical outcomes. Patient-reported outcomes, such as time to recover or quality-adjusted life-years (QALYs) could not be collected as this was a retrospective study. However, clinical outcomes can be assumed to reflect the time to recover to some degree. However, whenthe time to recover or QALYs were obtained VBAC performed better than ERCS. ${ }^{24}$ The counter-argument for this is that similar complication rates among the two groups would result in a similar time to recover and QALYs as complications are an indirect predictor of convalescence. The future pregnancy outcomes are also important to consider as VBAC and ERCS have significantly different effects. ERCS raises the likelihood of future cesarean sections, placenta previa, and morbidly adherent placenta whilst lowering the risk of pelvic floor disorders in later life. ${ }^{10,13}$ When the lifetime cost-effectiveness was compared using Markov models for long-term maternal outcomes, VBAC was found to be more cost-effective. ${ }^{24}$ The long-term implications of neonatal

Table 2: Comparison of outcomes between vaginal birth after cesarean section group and elective repeat cesarean section group

\begin{tabular}{|c|c|c|c|}
\hline Outcomes & $\operatorname{VBAC}(n=67)$ & $\operatorname{ERCS}(n=158)$ & Significance $(p)$ \\
\hline Postpartum hospital stay (days) [median, (IQ1-IQ3)] & $2(1-3)$ & $2(2-2.25)$ & $0.15^{\#}$ \\
\hline APGAR [median, (IQ1-IQ3)] & $10(10-10)$ & $10(10-10)$ & $0.25^{\#}$ \\
\hline Any complication (maternal or neonatal) & $15(22.38 \%)$ & $46(29.11 \%)$ & $0.30^{+}$ \\
\hline Any maternal complication & $6(8.96 \%)$ & $8(5.06 \%)$ & $0.27^{\div \cdot}$ \\
\hline Any neonatal complication & $9(13.43 \%)$ & $42(26.58 \%)$ & $0.031^{+}$ \\
\hline \multicolumn{4}{|l|}{ Maternal complications } \\
\hline $\mathrm{PPH}$ & $3(4.48 \%)$ & $3^{*}(1.90 \%)$ & $0.37^{\div}$ \\
\hline Postpartum fever & $2(2.99 \%)$ & $4(2.53 \%)$ & $1.0^{:}$ \\
\hline Wound infection & $0(0 \%)$ & $2(1.27 \%)$ & $1.0^{*}$ \\
\hline 3rd-degree tear & $1(1.49 \%)$ & $0(0 \%)$ & 0.30 \\
\hline \multicolumn{4}{|l|}{ Neonatal complications } \\
\hline Respiratory distress & $3(4.48 \%)$ & $6^{\beta}(3.80 \%)$ & $0.73^{\bullet}$ \\
\hline Fever & $1(1.49 \%)$ & $12(7.60 \%)$ & $0.12^{:}$ \\
\hline Jaundice & $5^{a}(7.46 \%)$ & $25(15.82 \%)$ & $0.092^{+}$ \\
\hline Meconium aspiration & $1(1.49 \%)$ & $0(0 \%)$ & $0.30^{4}$ \\
\hline NICU admissions & $5(7.86 \%)$ & $14(8.86 \%)$ & $0.73^{+}$ \\
\hline Other & $0(0 \%)$ & $2^{\gamma}(1.27 \%)$ & $1.0^{\circ}$ \\
\hline
\end{tabular}

"Mann-Whitney U test;

+ Chi-square test;

"Fisher's exact test;

* $>1.5$ Lpostpartum hemorrhage;

a One case of cephalohematoma;

${ }^{\beta}$ One case of transient tachypnea of the newborn;

${ }^{\gamma}$ Breastfeeding issue, hypoglycemia; APGAR, appearance, pulse, grimace, activity, and respiration; PPH, postpartum hemorrhage; NICU, newborn intensive care unit 
VBAC vs ERCS: A Cost-consequence Study

Table 3: Comparison of costs between vaginal birth after cesarean section group and elective repeat cesarean section group

\begin{tabular}{|c|c|c|c|}
\hline Costs ${ }^{1, \#}$ & $\operatorname{VBAC}(n=67)$ & $\operatorname{ERCS}(n=158)$ & Significance $(p)^{*}$ \\
\hline $\begin{array}{l}\text { Imaging } \\
\text { [median, (IQ1-IQ3)] }\end{array}$ & $20(9-25)$ & $9(9-9)$ & $<0.001$ \\
\hline $\begin{array}{l}\text { Wages } \\
\text { [median, (IQ1-IQ3)] }\end{array}$ & $337(286-339)$ & $337(337-337)$ & 0.08 \\
\hline $\begin{array}{l}\text { Instruments } \\
\text { [median, (IQ1-IQ3)] }\end{array}$ & $16(7-19)$ & 19 (19-19) & $<0.001$ \\
\hline $\begin{array}{l}\text { Anesthesia costs } \\
\text { [median, (IQ1-IQ3)] }\end{array}$ & $0(0-11)$ & $11(11-11)$ & $<0.001$ \\
\hline $\begin{array}{l}\text { Medications } \\
\text { [median, (IQ1-IQ3)] }\end{array}$ & $5(3-5)$ & $4(4-4)$ & 0.40 \\
\hline $\begin{array}{l}\text { Investigations } \\
\text { [median, (IQ1-IQ3)] }\end{array}$ & $2(2-4)$ & $2(2-4)$ & 0.74 \\
\hline $\begin{array}{l}\text { Space costs } \\
\text { [median, (IQ1-IQ3)] }\end{array}$ & $55(39-71)$ & $44(44-55)$ & 0.53 \\
\hline $\begin{array}{l}\text { Utility costs } \\
\text { [median, (IQ1-IQ3)] }\end{array}$ & 32 (19-39) & $26(26-32)$ & 0.97 \\
\hline $\begin{array}{l}\text { Maternal costs } \\
\text { [median, (IQ1-IQ3)] }\end{array}$ & $467(419-522)$ & 459 (452-486) & 0.74 \\
\hline $\begin{array}{l}\text { Total costs } \\
\text { [median, (IQ1-IQ3)] }\end{array}$ & $476(420-566)$ & 470 (452-494) & 0.78 \\
\hline
\end{tabular}

+Costs reported in USD (1 USD = 182.73 LKR);

*Mann-Whitney U test

Table 4: Subgroup analysis of outcomes between successful vaginal birth after cesarean section, failed vaginal birth after cesarean section, and elective repeat cesarean section groups

\begin{tabular}{|c|c|c|c|c|}
\hline Outcomes & VBAC-failed $(n=32)$ & VBAC-successful $(n=35)$ & $\operatorname{ERCS}(n=158)$ & Significance $(p)$ \\
\hline $\begin{array}{l}\text { Postpartum hospital stay (days) } \\
\text { [median, (IQ1-IQ3)] }\end{array}$ & $2(2-3.75)$ & $2(1-3)$ & $2(2-2.25)$ & $<0.01^{*}$ \\
\hline APGAR [median, (IQ1-IQ3)] & $10(10-10)$ & $10(10-10)$ & $10(10-10)$ & $0.33^{*}$ \\
\hline Any complication (maternal or neonatal) & $7(21.9 \%)$ & $8(22.9 \%)$ & $46(29.1 \%)$ & $0.58^{+}$ \\
\hline Any maternal complication & $2(6.2 \%)$ & $4(11.4 \%)$ & $8(5.1 \%)$ & $0.38^{:}$ \\
\hline Any neonatal complication & $5(15.6 \%)$ & $4(11.4 \%)$ & $42(26.6 \%)$ & $0.09^{+}$ \\
\hline \multicolumn{5}{|l|}{ Maternal complications } \\
\hline $\mathrm{PPH}$ & $1(3.1 \%)$ & $2(5.7 \%)$ & $3^{*}(1.9 \%)$ & $0.25^{\circ}$ \\
\hline Postpartum fever & $1(3.1 \%)$ & $1(2.9 \%)$ & $4(2.5 \%)$ & $1.0^{:}$ \\
\hline Wound infection & $0(0 \%)$ & $0(0 \%)$ & $2(1.3 \%)$ & $1.0^{\circ}$ \\
\hline 3rd-degree tear & $0(0 \%)$ & $1(2.9 \%)$ & $0(0 \%)$ & 0.30 \\
\hline \multicolumn{5}{|l|}{ Neonatal complications } \\
\hline Respiratory distress & $1(3.1 \%)$ & $2(5.7 \%)$ & $6^{\beta}(3.8 \%)$ & $0.86^{\circ}$ \\
\hline Fever & $1(3.1 \%)$ & $0(0 \%)$ & $12(7.6 \%)$ & $0.21^{\div}$ \\
\hline Jaundice & $4^{a}(12.5 \%)$ & $1(2.9 \%)$ & $25(15.8 \%)$ & 0.24 \\
\hline Meconium aspiration & $1(3.1 \%)$ & $0(0 \%)$ & $0(0 \%)$ & 0.14 \\
\hline NICU admissions & $2(6.2 \%)$ & $3(8.6 \%)$ & $14(8.9 \%)$ & $0.87^{\circ \cdot}$ \\
\hline Other & $0(0 \%)$ & $0(0 \%)$ & $2^{\gamma}(1.3 \%)$ & $1.0^{:}$ \\
\hline
\end{tabular}

*Kruskal-Wallis test;

+ Chi-square test;

"Fisher's exact test; APGAR, appearance, pulse, grimace, activity, and respiration; PPH, postpartum hemorrhage; NICU, newborn intensive care unit a one case of cephalohematoma

${ }^{\beta}$ one case of transient tachypnea of the newborn

Ybreastfeeding issue, hypoglycemia 
Table 5: Subgroup analysis of costs between successful vaginal birth after cesarean section, failed vaginal birth after cesarean section, and elective repeat cesarean section groups

\begin{tabular}{lcccc}
\hline Costs $^{+}$ & VBAC-failed $(n=32)$ & VBAC-successful $(n=35)$ & ERCS $(n=158)$ & Significance $(p)^{*}$ \\
\hline Imaging [median, (IQ1-IQ3)] & $16(9-25)$ & $20(9-25)$ & $9(9-9)$ & $<0.001$ \\
Wages [median, (IQ1-IQ3)] & $337(337-556)$ & $286(255-339)$ & $737(337-337)$ & $<0.001$ \\
Instruments [median, (IQ1-IQ3)] & $19(16-19)$ & $7-14)$ & $19(19-19)$ & $<0.001$ \\
Anesthesia costs [median, (IQ1-IQ3)] & $11(11-11)$ & $3(0-0)$ & $4(4-4)$ & $<0.001$ \\
Medications [median, (IQ1-IQ3)] & $5(5-7)$ & $2(2-3)$ & $2(2-4)$ & 0.001 \\
Investigations [median, (IQ1-IQ3)] & $2(2-4)$ & $50(39-65)$ & $44(44-55)$ & 0.02 \\
Space costs [median, (IQ1-IQ3)] & $65(44-76)$ & $26(19-39)$ & $26(26-32)$ & $<0.01$ \\
Utility costs [median, (IQ1-IQ3)] & $39(26-45)$ & $427(361-462)$ & $459(452-486)$ & $<0.001$ \\
Maternal costs (USD) [median, (IQ1-IQ3)] & $505(476-716)$ & $427(361-483)$ & $470(452-494)$ & $<0.001$ \\
Total costs (USD) [median, (IQ1-IQ3)] & $519(476-809)$ & &
\end{tabular}

+Costs reported in USD (1 USD = 182.73 LKR);

*Kruskal-Wallis test

complications, such as respiratory distress, fever, and meconium aspiration are also equally important in terms of both quality of life and economic cost. However, it was not possible to quantify the impact of these long-term outcomes.

The primary outcome, postpartum hospital stay depends on individual clinical practice and the set times of discharge from the hospital. ${ }^{22}$ This may be the reason why ERCS, successful and failed VBAC all had a similar median hospital stay. The relatively short hospital stay for ERCS contradicts evidence from literature and may be a result stemming from the relatively higher cesarean section rates in Sri Lanka. ${ }^{4,21}$ Also, the difference of one day rather than a difference of half a day may be more clinically relevant.

This study looked at costs from a provider's perspective and not from a societal perspective which would have included both direct hospital-borne costs and indirect patient-borne costs. A payer perspective for costs was considered as it was not possible to obtain indirect costs borne by the patient as this was a retrospective study. However, a payer perspective is still relevant for a country like Sri Lanka where there is a free public health care system. As this was a cost-consequence analysis, the costs and the outcomes for the two groups were described separately. However, it was not as powerful as a cost-benefit analysis or a cost-effectiveness study as the outcomes were neither assigned a monetary value nor weighed against cost. ${ }^{25,26}$

The costs for neonatal care, i.e., phototherapy, the cost of NICU admission, and NICU ventilation costs per day were extrapolated from the private sector due to logistical and financial limitations. The labor cost for neonates not admitted to the NICU (post-natal ward) was also not considered separately. However, cost estimation accuracy may be sacrificed in favor of ease of data collection if the perceived differences are small. ${ }^{25,26}$

Despite subgroup analysis showing that there was a significant difference in postpartum hospital stay between successful VBAC, failed VBAC, and ERCS groups, its clinical significance was minimal. Subgroup analysis also showed that the cost of the successful VBAC group was less than ERCS with the failed VBAC group being more than ERCS. Although this was found to be statistically significant the difference between the three groups was minimal. The finding of the lowest cost being for the successful VBAC with the highest for failed VBAC was consistent with evidence reported in the literature. ${ }^{10,19,27}$
The VBAC group represents the average of these two groups as the analysis was in terms of intention-to-treat. Therefore, it becomes obvious that the cost is likely to hinge on the VBAC success rate. A study by Chung et al. found VBAC to be cost-effective at a success rate of $74 \% .{ }^{27}$ In this study, the material cost in the VBAC group was similar to the ERCS group at a VBAC success rate of $80 \%$ with a $95 \%$ degree of confidence. Sri Lanka has an overall cesarean section rate of $39.1 \%$, suggesting a low threshold for cesarean section. ${ }^{4}$ Therefore, the feasibility of achieving a VBAC success rate of $80 \%$ may be difficultdue to a lack of skilled staff andthe non-availability of continuous electronic fetal monitoring in most centers. ${ }^{10,28,29}$

As the cost-effectiveness of VBAC hinges on its success rate, an important aspect that needs to be considered is patient selection, i.e., selection of suitable patients and also when to terminate the trial. Whilst this is affected by individual practices that vary widely among obstetricians, some uniformity and benchmarking would be necessary to improve success rates above $80 \%$. $^{10,13,27}$

A randomized controlled trial is an ideal design to compare two treatment groups. However, this cannot be done for VBAC vs ERCS due to ethical and medico-legal implications. A randomized controlled trial has stringent inclusion and exclusion criteria. Therefore, an observational study was done with stringent inclusion and exclusion criteria. This would also overcome some of the limitations of randomized controlled trials as it is a real-life setting. ${ }^{30}$

\section{Conclusion and Clinical Significance}

The crucial decision is to give VBAC to women who are likely to succeed. This remains one of the most elusive decisions in obstetrics. The findings of this study may raise serious concerns with possible implications for clinicians and health policy stakeholders in low resource settings because although study findings would suggest all women with a previous cesarean section to have an ERCS, the absence of an improved outcome in VBAC in contrast to available evidence suggests suboptimal management of women with a previous cesarean section. 


\section{Compliance with Ethical Standards}

The ethical approval was obtained from the ethical review committee of the Faculty of Medicine, University of Kelaniya (P/139/07/2019). Informed consent was not required as the anonymized data for this observational study was extracted from patient records by a medically qualified person.

\section{Acknowledgments}

We are grateful to the staff of the professorial obstetrics and gynecology units of North Colombo Teaching Hospital, the Director, North Colombo Teaching Hospital, and Dr. Prageeth Thilakaratne, registrar of the unit.

\section{References}

1. Gibbons L, Belizán JM, Lauer JA, et al. The global numbers and costs of additionally needed and unnecessary caesarean sections performed per year: overuse as a barrier to universal coverage. World Health Report2010; Background Paper, 30.

2. Betrán $A P, Y e J$, Moller $A B$, et al. The increasing trend in caesarean section rates: global, regional and national estimates: 1990-2014. PLoS One 2016;11(2):e0148343. DOI:10.1371/journal.pone.0148343.

3. MenackerF,DeclercqE,MacdormanMF.Cesarean delivery:background, trends, and epidemiology. SeminPerinatol2006;30(5):235-241. DOI: 10.1053/j.semperi.2006.07.002.

4. Family Health Bureau. Indicators [FHB website]. 2018. http://fhb. health.gov.lk/index.php/en/statistics. Accessed March 31, 2020.

5. Gunasekara PC, Wijesinghe PS, Goonewardene I.The caesarean section rate is rising. Ceylon Med J2001;46(4):147-150. DOI: 10.4038/ cmj.v46i4.6466.

6. Luce A, Cash M, Hundley V, et al. "Is it realistic?" the portrayal of pregnancy and childbirth in the media. BMC Pregnancy Childbirth 2016;16:40. DOI: 10.1186/s12884-016-0827-x.

7. Ji H, Jiang $H$, Yang $L$, et al. Factors contributing to the rapid rise of caesarean section: a prospective study of primiparous Chinese women in Shanghai. BMJ Open 2015;5(11):e008994. DOI: 10.1136/ bmjopen-2015-008994.

8. Feng $\mathrm{XL}, \mathrm{Xu} \mathrm{L}, \mathrm{Guo} Y$, et al. Factors influencing rising caesarean section rates in China between 1988 and 2008. Bull World Health Organ 2012;90(1):30-39. DOI: 10.2471/BLT.11.090399.

9. Mittal S, Pardeshi S, Mayadeo N, et al. Trends in caesarean delivery: rate and indications. J ObstetGynaecol India 2014;64(4):251-254. DOI: 10.1007/s13224-013-0491-2.

10. Royal College of Obstetricians and Gynecologists. Green top guideline no. 45. (October 2015). Birth after previous caesarean birth.

11. American College of Obstetricians and Gynecologists. ACOG Practice Bulletin No. 205: vaginal birth after cesarean delivery. Obstet Gynecol 2019;133(2):e110-e127. DOI: 10.1097/AOG.0000000000003078.

12. Goonewardene M,Peiris $M$, Kariyawasam S, et al.Analysis of high caesarean section rates - the second step after audits using the Ten Group Classification System. Ceylon Med J2017;62(3):149-158. DOI: 10.4038/cmj.v62i3.8518.

13. Scott JR. Intrapartum management of trial of labour after caesarean delivery: evidence and experience. BJOG 2014;121(2):157-162. DOI:10.1111/1471-0528.12449.
14. Fawsitt CG, Bourke J, Greene RA, et al. At what price? A costeffectiveness analysis comparing trial of labor after previous caesarean versus elective repeat caesarean delivery. PLoS One 2013;8(3):e58577. DOI:10.1371/journal.pone.0058577.

15. Fobelets M, Beeckman K, Faron G, et al. Vaginal birth after caesarean versus elective repeat caesarean delivery after one previous caesarean section: a cost-effectiveness analysis in four European countries. BMC Pregnancy Childbirth 2018;18:92. DOI:10.1186/s12884018-1720-6.

16. Rogers AJ, Rogers NG, Kilgore ML, et al. Economic evaluations comparing a trial of labor with an elective repeat cesarean delivery: a systematic review. Value Health 2017;20:163-173. DOI:10.1016/j. jval.2016.08.738.

17. Ekanayake C, Pathmeswaran A, Kularatna S, et al. Challenges of costing a surgical procedure in a lower-middle-income country. World J Surg 2019;43(1):52-59. DOI: 10.1007/s00268-018-4773-9.

18. Campbell Collaboration. CCEMG-EPPI-Centre Cost Converter [University College London Website]. 2019. https://eppi.ioe.ac.uk/ costconversion/.

19. Friedman AM, Ananth CV, Chen L, et al. An economic analysis of trial of labor after cesarean delivery. J Matern Fetal Neonatal Med 2016;29(7):1030-1035. DOI:10.3109/14767058.2015.1035250.

20. Royal Australian and New Zealand College of Obstetricians and Gynecologists. (March 2019). Birth after previous caesarean section.

21. Devkare V, Agarwal N, Gayakwad N, et al. Maternal and fetal outcome of VBAC after first previous LSCS in a tertiary care teaching hospital of Western India. Int J Curr Res Med Sci 2017;3(7):8-17.DOI: 10.22192/ ijcrms.2017.03.07.002.

22. Krell RW, Girotti ME, Dimick JB. Extended length of stay after surgery: complications, inefficient practice, or sick patients?JAMA Surg 2014;149(8):815-820. DOI:10.1001/jamasurg.2014.629.

23. Mirteymouri $M$, Ayati S, Pourali $L$, et al. Evaluation of maternalneonatal outcomes in vaginal birth after cesarean delivery referred to maternity of academic hospitals. J Family Reprod Health 2016;10(4):206-210.

24. Gilbert SA, Grobman WA, Landon MB, et al. Lifetime cost-effectiveness of trial of labor after cesarean in the United States. Value Health 2013;16(6):953-964. DOI:10.1016/j.jval.2013.06.014.

25. Drummond $M$, Sculpher $M$, Torrance $G$, et al. Methods for the Economic Evaluation of Health Care Programme. Oxford: Oxford University Press; 2005.

26. Gray A, Clarke P, Wolstenholme J, et al. Applied Methods of Costeffectiveness Analysis in Health Care. Oxford: Oxford University Press; 2011.

27. Chung A, Macario A, El-Sayed YY, et al. Cost-effectiveness of a trial of labor after previous caesarean. ObstetGynecol 2001;97(6):932-941. DOI:10.1016/s0029-7844(01)01355-2.

28. Kalisa R, Rulisa S, van Roosmalen J, et al. Maternal and perinatal outcome after previous caesarean section in rural Rwanda. BMC Pregnancy Childbirth 2017;17(1):272. DOI:10.1186/s12884017-1467-5.

29. Housseine N, Punt MC, Browne JL, et al. Delphi consensus statement on intrapartum fetal monitoring in low-resource settings. Int J Gynaecol Obstet 2019;146(1):8-16. DOI:10.1002/ijgo.12724.

30. Booth CM, Tannock IF. Randomised controlled trials and populationbased observational research: partners in the evolution of medical evidence. Br J Cancer 2014;110(3):551-555. DOI:10.1038/ bjc.2013.725. 